\title{
A systematic review of perioperative seizure prophylaxis during brain tumor resection: the case for a multicenter randomized clinical trial
}

\author{
Vyshak Chandra, MS, ${ }^{1}$ Andrew K. Rock, MHS, MS, ${ }^{1}$ Charles Opalak, MD, MPH, ${ }^{1}$ \\ Joel M. Stary, MD, PhD, ${ }^{1}$ Adam P. Sima, PhD, ${ }^{2}$ Matthew Carr, BS, ${ }^{1}$ Rafael A. Vega, MD, PhD, ${ }^{1}$ and \\ William C. Broaddus, MD, PhD ${ }^{1}$ \\ 1Department of Neurosurgery, Virginia Commonwealth University Health System, Medical College of Virginia; and ${ }^{2}$ Department \\ of Biostatistics, Virginia Commonwealth University, Richmond, Virginia
}

\begin{abstract}
OBJECTIVE The majority of neurosurgeons administer antiepileptic drugs (AEDs) prophylactically for supratentorial tumor resection without clear evidence to support this practice. The putative benefit of perioperative seizure prophylaxis must be weighed against the risks of adverse effects and drug interactions in patients without a history of seizures. Consequently, the authors conducted a systematic review of prospective randomized controlled trials (RCTs) that have evaluated the efficacy of perioperative seizure prophylaxis among patients without a history of seizures.
\end{abstract}

METHODS Five databases (PubMed/MEDLINE, Cochrane Central Register of Controlled Trials, CINAHL/Academic Search Complete, Web of Science, and ScienceDirect) were searched for RCTs published before May 2017 and investigating perioperative seizure prophylaxis in brain tumor resection. Of the 496 unique research articles identified, 4 were selected for inclusion in this review.

RESULTS This systematic review revealed a weighted average seizure rate of $10.65 \%$ for the control groups. There was no significant difference in seizure rates among the groups that received seizure prophylaxis and those that did not. Further, this expected incidence of new-onset postoperative seizures would require a total of 1258 patients to enroll in a $\mathrm{RCT}$, as determined by a Farrington-Manning noninferiority test performed at the 0.05 level using a noninferiority difference of $5 \%$.

CONCLUSIONS According to a systematic review of major RCTs, the administration of prophylactic AEDs after brain tumor resection shows no significant reduction in the incidence of seizures compared with that in controls. A large multicenter randomized clinical trial would be required to assess whether perioperative seizure prophylaxis provides benefit for patients undergoing brain tumor resection.

https://thejns.org/doi/abs/10.3171/2017.8.FOCUS17442

KEY WORDS brain neoplasms; neurosurgery; seizures; anticonvulsants; prevention and control; systematic review

$\mathrm{S}$ EIZURES are debilitating and burdensome for the health care system. Patients with seizures have higher medical expenses, lower employment rates, and barriers to social participation. ${ }^{11}$ Seizures lead to longer hospital stays and increased health care costs. The risk of new-onset seizure for a patient with a brain tumor is reported to be $20 \%-90 \% .^{7,12,20}$ Postcraniotomy seizure rates for patients with no prior history of seizure range from $7 \%$ to $18 \%{ }^{4,5}$ Antiepileptic drugs (AEDs) have been used to decrease the rate of postoperative seizures. This prac- tice was first studied in the 1980s, with investigators in a double-blind trial concluding that preoperative phenytoin administration decreased seizure rates by $50 \%: 2^{20}$ however, the study included craniotomies for a variety of pathologies, and there was no significant difference in seizure rates between the AED-treated and untreated supratentorial tumor resection groups.

A 2015 survey of surgeons revealed that more than 63\% of them administer seizure prophylaxis after tumor resection in patients without a history of seizures. ${ }^{3}$ Eighty-five 
percent of the surgeons who practice prophylaxis reported that they use the antiepileptic levetiracetam. The duration of prophylaxis varied from 1 week to more than 6 weeks. This suggests that a significant minority of tumor surgeons do not endorse the perioperative use of AEDs in seizure-naïve patients. This survey also revealed that $82 \%$ of the respondents would guide their future practice in this area based on the results of a well-designed randomized controlled trial (RCT).

There is no Class I evidence to support the widespread practice of administering prophylactic AEDs in patients undergoing tumor resection. Further, pharmaceuticals are not benign. Levetiracetam can cause behavioral problems (anger, apathy, irritability, neurosis), headache, drowsiness, suicidal ideation, increased blood pressure, and gastrointestinal disturbances. Phenytoin has a larger set of side effects, such as atrial conduction depression, arrhythmias, cerebral atrophy, cerebral dysfunction, peripheral neuropathy, dermatological issues, and various immunological issues. The total direct cost of a 7-day seizure prophylaxis with intravenous levetiracetam is $\$ 8784.63$ and with intravenous phenytoin $\$ 8743.78$. The cost-effectiveness ratio per successful seizure prophylaxis regimen (SSPR) was shown to be $\$ 10,044.91$ for intravenous levetiracetam, compared with $\$ 11,525.63$ for intravenous phenytoin. ${ }^{12}$

Given that the perioperative administration of AEDs is one of the most common practices encountered in neurological intensive care patients, we conducted a systematic review of relevant studies of perioperative seizure prophylaxis in patients undergoing tumor resection. Additionally, we provide an outline of the trial that would be necessary to provide Class I evidence. This is both a knowledge update and a blueprint for moving forward.

\section{Methods \\ Literature Search and Inclusion Criteria}

This systematic review was conducted according to the Preferred Reporting Items for Systematic Reviews and Meta-Analyses for Protocols (PRISMA-P) guidelines. ${ }^{18}$ Five databases were searched between May 10 and May 13, 2017. The databases included PubMed/MEDLINE, Cochrane Central Register of Controlled Trials (CENTRAL), CINAHL/Academic Search Complete, Web of Science, and ScienceDirect. Searches used combinations of specific terms related to neurosurgery, brain neoplasm, anticonvulsants, and prophylaxis (details of search strategies are provided in the Appendix). Only original peer-reviewed randomized clinical trials in humans whose results were published in the English language were considered for inclusion. In addition, the references of previous systematic reviews, meta-analyses, and selected studies were evaluated for the inclusion of additional studies.

\section{Data Extraction and Outcome Measures}

One reviewer (A.K.R.) evaluated and selected articles by title, abstract, and full-text review for data abstraction. Abstracted data included first author, year, location, study design, sample size, sample characteristics, tumor type, AED type, control group, timing of intervention, duration of intervention, follow-up interval, outcome definition, seizure incidence, risk estimate, and conclusions. The risk of bias (low, high, uncertain) of included trials was assessed using the Cochrane Handbook for Systematic Reviews of Interventions ${ }^{10}$ and the following 6 domains: random sequence generation, allocation concealment, blinding, incomplete outcome data, selective reporting, and other bias. The weighted average seizure rate of untreated controls was calculated based on reported values from the included studies. Power analyses were conducted using the weighted average seizure rate to estimate the number of patients required to demonstrate noninferiority within an RCT. All power analyses were based on the FarringtonManning noninferiority test performed at the 0.05 level using a noninferiority difference of $5 \%$.

\section{Results \\ Study Selection}

A total of 496 unique articles were identified through the database searches and underwent title, abstract, and full-text review (Fig. 1). After screening by title, there were 84 articles that underwent abstract review. Sixtyeight articles were subsequently excluded, and 16 articles were considered eligible for full-text review. Following the full-text review, 13 articles were excluded. Reasons for exclusion comprised a lack of perioperative seizure outcomes, ${ }^{15,16}$ a heterogeneous sample of neurosurgery patients, ${ }^{9}$ non-English language,,${ }^{19,23}$ absent assessment of seizure prophylaxis for brain tumor resection, $7,22,28$ and study design other than RCT. ${ }^{13,14,21,24,25}$ One additional study was identified through a review of the reference lists. A total of 4 studies met inclusion criteria and were considered in this systematic review (Table 1). Of these trials, one compared prophylactic phenobarbital or phenytoin to no intervention, ${ }^{8}$ one compared prophylactic phenytoin to no intervention, ${ }^{27}$ one compared prophylactic gabapentin to phenytoin use, ${ }^{26}$ and one evaluated the addition of prophylactic phenytoin to current AED therapy. ${ }^{2}$

\section{Assessment of Risk of Bias for the Included Studies}

Details from the assessment of risk of bias for included studies are presented in Fig. 2. Despite claims of being RCTs, only 1 study reported methodology in support of random sequence generation when allocating participants to AED and control groups. Although 2 of the studies blinded researchers for outcome assessment, none of the studies had allocation concealment or blinded participants and personnel to allocated interventions. One study had incomplete data for outcomes because the trial was terminated after interim futility analysis had demonstrated that the power would be too low to show superiority between the AED and control groups.

\section{Seizure Outcomes}

Four clinical trials assessed perioperative AED prophylaxis for preventing seizures in patients undergoing resection of brain tumors (Table 1), ${ }^{2,8,26,27}$ All of the studies included heterogeneous samples of patients with supratentorial tumors within a variety of anatomical locations. Two trials compared AED prophylaxis to no intervention, ${ }^{8,27}$ 


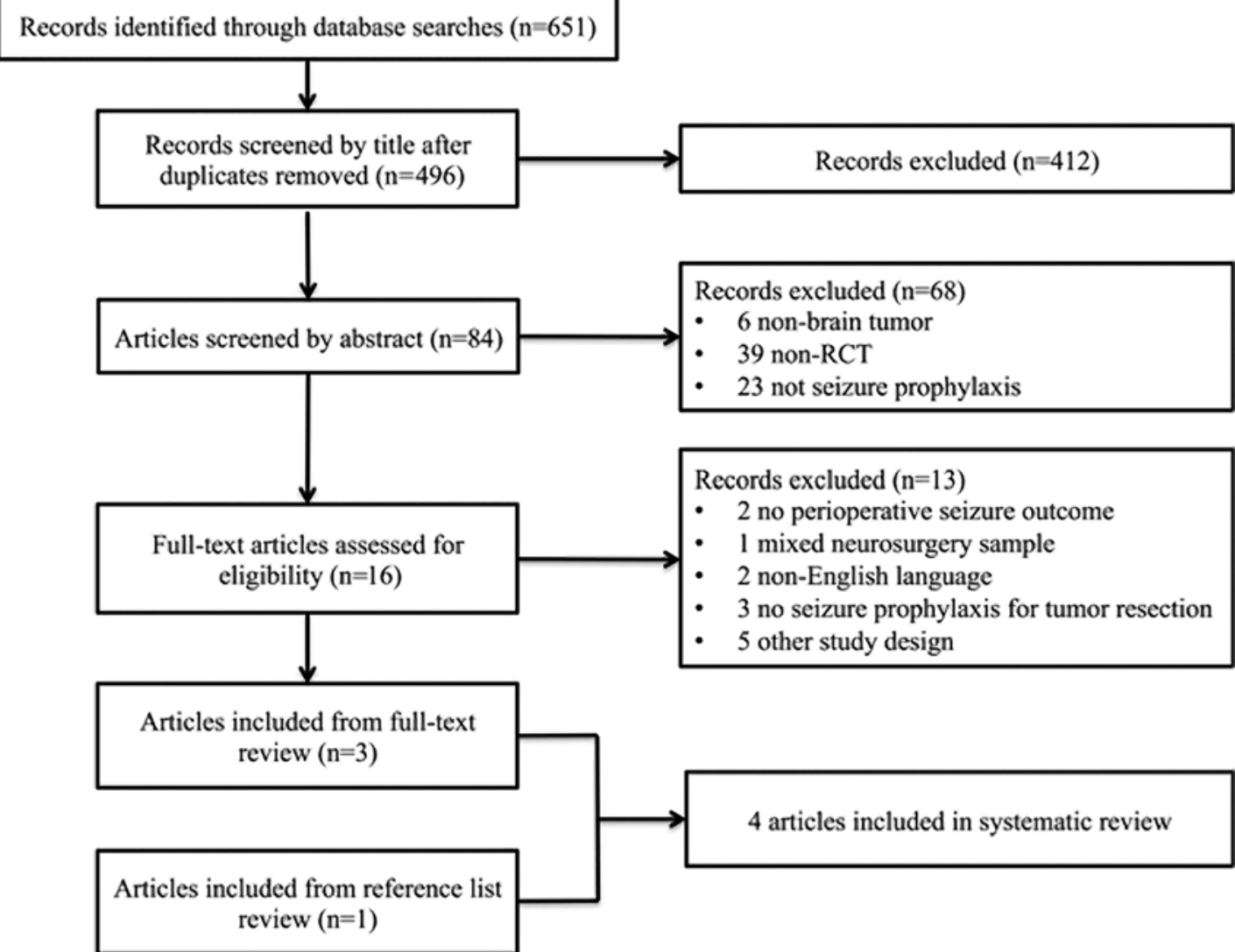

FIG. 1. Flow diagram according to the PRISMA-P statement.

while the other 2 trials compared the addition of phenytoin to current AED therapy ${ }^{2}$ and gabapentin to treatment with phenytoin, respectively. ${ }^{26}$ There was significant diversity across studies in the timing of the intervention, duration of intervention, follow-up interval, and outcome definition. One study did not specify the timing and duration of the intervention. ${ }^{8}$ Two studies initiated AED therapy intraoperatively and continued treatment for 7 days, ${ }^{2,27}$ while 1 study initiated AED therapy 7 days prior to surgery and continued treatment for 6-12 months for meningiomas or indefinitely for glial tumors. ${ }^{26}$ The follow-up interval across studies ranged from 7 days to 12 months. Early postoperative seizures were defined by a cutoff of $\leq 7$ days in 2 studies ${ }^{2,8}$ and $\leq 30$ days in another study ${ }^{27}$ Additional outcomes included late seizures ( $>7$ days) and a history of any new seizure activity. Only 1 study had a neurologist blinded to patient treatment who evaluated and confirmed the diagnosis of seizures on clinical grounds or electroencephalography. ${ }^{27}$ Across all of the studies, there was no significant reduction in the incidence of seizures among those in the intervention groups compared with controls.

Franceschetti et al. randomly assigned 63 patients without preoperative seizures to receive phenobarbital $(n=25)$, phenytoin $(\mathrm{n}=16)$, or no medication $(\mathrm{n}=22) .{ }^{8}$ Early postoperative seizures ( $\leq 7$ days) occurred in $4(18 \%)$ of 22 untreated patients and $3(7 \%)$ of the 41 patients treated with phenobarbital or phenytoin, whereas late postoperative seizures ( $>7$ days) occurred in 3 (21\%) of 14 untreated patients and $3(12 \%)$ of 25 patients treated with phenobarbital or phenytoin. Seizure incidence did not significantly differ between groups. De Santis et al. conducted a prospective open-label RCT to determine the potential effectiveness of phenytoin in addition to a patient's current AED therapy in preventing early postoperative seizures in those undergoing craniotomy for supratentorial brain tumor. ${ }^{2}$ Patients randomly assigned to the phenytoin group were treated intraoperatively with a loading dose of 18 $\mathrm{mg} / \mathrm{kg}$ followed by 7 days of treatment adjusted according to serum concentrations. Early postoperative seizures occurred in $13 \%$ of patients in the phenytoin group and $11 \%$ of patients in the control group. The incidence of early postoperative seizures did not differ between prophylaxis and control groups. Türe et al. evaluated the postoperative effectiveness of gabapentin on acute postoperative pain as compared with phenytoin for AED prophylaxis in patients undergoing craniotomy for supratentorial tumor resection. ${ }^{26}$ Seizure activity was assessed at 0,15 , and 30 minutes; 1, 2, 4, 6, 12, 24, and 48 hours; and 1 month postoperatively. Only 1 patient in the phenytoin group had a seizure on postoperative Day 3.

$\mathrm{Wu}$ et al. pursued a prospective RCT examining the perioperative use of prophylactic AEDs (phenytoin) in patients without a history of seizures. Their study was designed to accrue 142 patients using an estimated seizure incidence of $30 \%$ in the control group and $10 \%$ in the prophylaxis group. After 6 years, the study was closed after 
123 patients had been enrolled and an independent interim futility analysis had demonstrated a low likelihood that either the observation or phenytoin prophylaxis group would be superior using the full sample size. At the time, early seizures were observed in only $8 \%$ of the control group, and the true power to detect a two-thirds reduction in postsurgical 30-day seizure incidence using 123 patients was $19 \%$. The probability that there would be insufficient evidence at the end of the trial to show superiority was 0.997 , and calculations using the lower-than-expected incidence of seizures of $8 \%$ demonstrated that over 700 patients would be needed to detect the anticipated difference. Among the 123 patients who were enrolled, the incidence of overall, early ( $\leq 30$ days), late ( $>30$ days), and clinically significant seizures was $24 \%, 10 \%, 14 \%$, and $3 \%$ for the prophylaxis group and $18 \%, 8 \%, 10 \%$, and $2 \%$ for controls, respectively. There was no significant difference in seizure incidence between the control and prophylaxis groups. ${ }^{27}$

The weighted average seizure rate was $10.65 \%$ based on reported values from control groups. Results from power analyses estimating the sample size for a noninferiority $\mathrm{RCT}$ are presented in Table 2.

TABLE 1. A comparison of RCTs assessing perioperative AED prophylaxis in patients undergoing brain tumor resection

\begin{tabular}{|c|c|c|c|c|}
\hline Parameter & Franceschetti et al., 1990 & De Santis et al., 2002 & Türe et al., 2009* & Wu et al., $2013 \dagger$ \\
\hline Location & Italy & Italy & Turkey & USA \\
\hline Study design & RCT & Open-label RCT & RCT & RCT \\
\hline Sample size & $63 \ddagger$ & 200 & 75 & 123 \\
\hline $\begin{array}{l}\text { Sample charac- } \\
\text { teristics }\end{array}$ & $\begin{array}{l}34 \mathrm{M}, 29 \mathrm{~F} \text {; mean age: } \\
55 \text { yrs }\end{array}$ & $\begin{array}{l}103 \text { M, } 97 \text { F; preop Szs: 67; } \\
\text { preexisting AED therapy: } 185\end{array}$ & $36 \mathrm{M}, 39 \mathrm{~F}$ & $67 \mathrm{M}, 56 \mathrm{~F}$ \\
\hline Tumor type & $\begin{array}{l}\text { Supratentorial neoplasm } \\
\text { location: frontal, } 12 ; \\
\text { temporal, } 18 ; \text { central, } \\
\text { 2; parietal, } 16 ; \text { occipi- } \\
\text { tal, } 15 \text {; meningiomas: } \\
\text { 27; malignant glial: } 23 ; \\
\text { metastases: } 13\end{array}$ & $\begin{array}{l}\text { Supratentorial tumor location: } \\
\text { frontal, } 70 \text {; temporal, } 47 ; \\
\text { parietal, } 41 \text {; sellar, 17; oc- } \\
\text { cipital, } 7 \text {; deep structures, } 6 \text {; } \\
\text { intraventricular, 6; tentorial, 6; } \\
\text { glioma: } 95 ; \text { meningioma: } 81 ; \\
\text { metastases: 10; other: } 14\end{array}$ & $\begin{array}{l}\text { Supratentorial tumor } \\
\text { surgical route: fron- } \\
\text { tal, 17; parietal, } \\
\text { 6; temporal, 4; } \\
\text { pterional, 48; menin- } \\
\text { gioma: } 32 \text {; glioma: } \\
\text { 42; metastases: } 1\end{array}$ & $\begin{array}{l}\text { Intraparenchymal supratentorial tumor loca- } \\
\text { tion: frontal, 48; temporal, 34; parietal, } \\
\text { 17; occipital, 18; other, 6; metastases: } 77 \text {; } \\
\text { glioma: } 46\end{array}$ \\
\hline AED type & $\mathrm{PB}$ or $\mathrm{PHT}$ & PHT plus current AED therapy & Gabapentin & PHT \\
\hline Control group & No medication & Current AED therapy & PHT & No medication \\
\hline $\begin{array}{l}\text { Timing of } \\
\text { intervention }\end{array}$ & NS & Intraoperatively & 7 days before surgery & Intraoperatively \\
\hline $\begin{array}{l}\text { Duration of } \\
\text { intervention }\end{array}$ & NS & 7 days & $\begin{array}{l}\text { 6-12 mos for meningio- } \\
\text { mas or indefinitely } \\
\text { for glial tumors }\end{array}$ & Tapered on POD 8 \\
\hline FU interval & Minimum of 6 mos & 7 days & $1 \mathrm{mo}$ & 12 mos \\
\hline $\begin{array}{l}\text { Outcome } \\
\text { definition }\end{array}$ & $\begin{array}{l}\text { Early ( } \leq 7 \text { days) \& late } \\
\text { postop Szs }\end{array}$ & Early postop Szs ( $\leq 7$ days) & $\begin{array}{l}\text { Sz activity at } 0,15,30 \\
\text { mins, } 1,2,4,6,12 \\
24,48 \mathrm{hrs}, \& 1 \mathrm{mo} \\
\text { postoperatively }\end{array}$ & $\begin{array}{l}\text { Early Sz ( } \leq 30 \text { days) or clinically significant } \\
\text { Sz w/ neurological deficits assessed } \\
\text { daily for } 3 \text { days after surgery, on POD 8, } \\
\text { \& every } 2-3 \text { mos for up to } 12 \text { mos }\end{array}$ \\
\hline Sz incidence & $\begin{array}{l}\text { Early Szs in } 18 \% \text { untreat- } \\
\text { ed \& } 7 \% \text { PB- or PHT- } \\
\text { treated; late Szs in } \\
21 \% \text { untreated \& } 12 \% \\
\text { PB- or PHT-treated }\end{array}$ & $\begin{array}{l}\text { Early Szs in } 13 \% \text { PHT-treated \& } \\
11 \% \text { controls }\end{array}$ & $\begin{array}{l}1 \text { patient in PHT group } \\
\text { had partial Sz on } \\
\text { POD 3; all patients } \\
\text { Sz free at 1-mo FU }\end{array}$ & $\begin{array}{l}\text { Szs in } 18 \% \text { controls \& } 24 \% \text { prophylaxis } \\
\text { group ( } p=0.51) \text {; early Szs ( } \leq 30 \text { days) in } \\
8 \% \text { controls \& } 10 \% \text { prophylaxis group ( } p \\
\text { = } 1.00) \text {; late Szs }(>30 \text { days) in } 10 \% \text { con- } \\
\text { trols \& } 14 \% \text { prophylaxis group ( } p=1.00 \text { ); } \\
\text { clinically significant Szs in } 2 \% \text { controls \& } \\
3 \% \text { prophylaxis group }(p=0.62)\end{array}$ \\
\hline Risk estimate & NS & NS & NS & $\begin{array}{l}\text { Odds of early Sz in prophylaxis vs control } \\
\text { was } 1.4(95 \% \mathrm{Cl} 0.425-4.763)\end{array}$ \\
\hline $\begin{array}{l}\text { Statistical } \\
\text { significance }\end{array}$ & No & No & No & No \\
\hline $\begin{array}{l}\text { Authors support } \\
\text { prophylactic } \\
\text { AED use }\end{array}$ & Yes & Noß & No & No \\
\hline
\end{tabular}

FU = follow-up; NS = not specified; PB = phenobarbital; PHT = phenytoin; $\mathrm{POD}=$ postoperative day; $\mathrm{Sz}$ = seizure; USA = United States of America.

* Study designed to assess postoperative analgesic efficacy of gabapentin compared to phenytoin.

† Trial closed early due to limited power to observe significant differences between groups.

$\ddagger$ After removing patients with preoperative seizures.

$\S$ Conclusions only extend to short-term PHT prophylaxis plus current AED therapy. 




FIG. 2. Risk of bias summary for each included study. Green plus sign indicates low risk; red minus sign, high risk; and yellow question mark, not reported.

\section{Discussion \\ Clinical Implications}

We conducted a systematic review of RCTs investigating perioperative seizure prophylaxis in patients without a history of seizures who were undergoing brain tumor resection, including data from 4 studies. ${ }^{2,8,26,27}$ Our review suggests that prophylactic AED administration may not be effective in preventing seizures in the perioperative period in patients without a history of seizures. Across all of the included studies, there was no significant difference in seizure incidence in the intervention groups compared with controls. Without clear evidence of improved seizure control, neurosurgeons must reconsider the routine perioperative administration of AEDs in patients without a history of seizures who are undergoing brain tumor resection. Apparently, a sizable minority of our colleagues has already done so. ${ }^{8}$

\section{Limitations of Current Evidence}

Current evidence has sizable limitations. Particularly striking is the lack of any trial evaluating levetiracetam. The reviewed trials investigated gabapentin, phenytoin, and phenobarbital. Levetiracetam is, by far, the AED most commonly used by tumor neurosurgeons, and its sideeffect profile compares favorably to that for older AEDs. In addition, the reviewed studies did not consistently report tumor location and pathology, despite a strong belief among tumor surgeons about the varying degrees of epileptogenicity based on pathology. Nor was seizure timing or optimal length of prophylaxis consistently specified. These are major limitations within the existing RCTs that have investigated perioperative seizure prophylaxis for brain tumor resection.

\section{Future Directions}

There is widespread consensus that an RCT is needed
TABLE 2. Expected sample size required for a noninferiority study aimed at demonstrating similar seizure rates for prophylaxis and control groups

\begin{tabular}{ccccc}
\hline \multicolumn{3}{c}{ Sz Rate } & & \\
\cline { 1 - 2 } AED Group & Control Group & Total No. & No. of Sites ${ }^{*}$ \\
\cline { 1 - 2 } $10 \%$ & $14 \%$ & 26,092 & 131 \\
\hline $10 \%$ & $13 \%$ & 6,322 & 32 \\
\hline $10 \%$ & $12 \%$ & 2,720 & 14 \\
\hline $10 \%$ & $11 \%$ & 1,480 & 8 \\
\hline $10 \%$ & $10.65 \%$ & 1,258 & 7 \\
\hline $10 \%$ & $10 \%$ & 914 & 5 \\
\hline $10 \%$ & $9 \%$ & 612 & 4 \\
\hline $10 \%$ & $8 \%$ & 434 & 3 \\
\hline
\end{tabular}

This information is based on a Type I error $=0.05$, power $=80 \%$, difference $=$ $5 \%$.

* Number of sites calculated from an accrual rate of 50 per site each year for 4 years.

to provide solid grounds for or against antiseizure prophylaxis in tumor surgery. Given the consistent lack of differences found between prophylaxis and control groups, the objective moving forward should not be to determine differences between these 2 groups (that is, superiority), but rather to show that these groups do not have different seizure profiles (that is, noninferiority). Table 2 shows the required sample size for a noninferiority study to achieve $80 \%$ power in comparing a prophylactic AED group to a control group in which the AED group has a $10 \%$ seizure rate and the control group has a seizure rate that varies from $8 \%$ to $14 \%$. All power analyses were based on the Farrington-Manning noninferiority test performed at the 0.05 level using a noninferiority difference of $5 \%{ }^{6}$

To estimate possible enrollment, we extracted data on all of our institution's first-time supratentorial craniotomies in adults for the period of 2014-2015. One hundred ninety-seven cases of first-time supratentorial tumor resection were performed during this period. The current literature suggests an enrollment rate slightly above 50\% among eligible cancer patients approached for RCTs. ${ }^{17}$ Therefore, it is estimated that at our center, approximately 50 patients a year could be accrued to a possible AED RCT. This is clearly too few to expect a well-powered study conducted in a timely manner; therefore, such a trial would undoubtedly require multiple centers. To estimate the number needed, a guide is given but we assumed a 4 -year accrual of 50 patients per site per year. These values should only be used as a guide as they do not incorporate any intersite variation. It is possible that including sites with larger patient volumes could significantly reduce the number of sites required for such a study.

We propose that such a trial would compare levetiracetam to placebo, with accrual occurring over a 4-year period. Levetiracetam would be chosen since its use reflects current practice; it is associated with similar efficacy and fewer adverse effects compared to other anticonvulsants. Outcome measures would include seizure incidence at 7 and 30 days postoperatively. Prophylaxis would be administered at the time of surgery and continued for 7 days 
postoperatively in accordance with the most commonly reported practice. Seizures would be assessed through patient, family, and practitioner reporting. All participants would be blinded to the patient treatment arm. Careful attention would be paid to monitoring and recording other adverse events in order to capture those related to AED administration. Costs of such a trial would be significant and would include the course of levetiracetam or placebo, as well as the staff for handling data and conducting follow-up. However, the cost would compare favorably with those for other RCTs since levetiracetam is now a generic drug, and the investigators could integrate existing clinical protocols and information gathering to reduce costs.

\section{Conclusions}

The question of whether to use perioperative seizure prophylaxis for patients undergoing brain tumor resection is unresolved. The use of AEDs postoperatively has become routine across major academic centers worldwide despite evidence indicating no significant reduction in the incidence of seizure among patients who receive prophylactic AEDs. Remarkably, the most commonly used AED, levetiracetam, has not been studied for this application in an RCT. There are significant limitations in the available evidence, as outlined above. To determine with any confidence and statistical significance that there is noninferiority in the seizure rate when giving or withholding AEDs, a large multicenter RCT should be performed.

\section{Appendix}

\section{PubMed/MEDLINE Search Strategy}

("Neurosurgery"[Mesh] OR "Neurosurgical Procedures"[Mesh] OR "General Surgery"[Mesh] OR "Surgical Procedures, Operative"[Mesh] OR "surgery" [Subheading] OR brain surgery OR craniectomy OR neurological surgery OR craniotomy OR neurosurgery $\mathrm{OR}$ operation $\mathrm{OR}$ operative $\mathrm{OR}$ operative surgical procedure* OR procedure* OR resection OR surgery OR surgical OR surgical procedure* OR general surgery) AND ("Brain Neoplasms"[Mesh] OR brain tumor OR brain neoplasm*) AND ("Seizures"[Mesh] OR "Epilepsy, Partial, Sensory"[Mesh] OR "Epilepsy, Tonic-Clonic"[Mesh] OR "Epilepsy, Partial, Motor"[Mesh] OR "Epilepsy, Generalized"[Mesh] OR "Epilepsy, Post-Traumatic"[Mesh] OR "Epilepsies, Partial”[Mesh] OR "Epilepsy, Absence"[Mesh] OR "Epilepsies, Myoclonic"[Mesh] OR "Epilepsy”[Mesh] OR "Epilepsy, Complex Partial”[Mesh] OR "Anticonvulsants"[Mesh] OR "Anticonvulsants" [Pharmacological Action] OR "Barbiturates"[Mesh] OR "Propofol"[Mesh] OR "Hydantoins"[Mesh] OR "Carbamazepine"[Mesh] OR "brivaracetam" [Supplementary Concept] OR "clobazam" [Supplementary Concept] OR "Clonazepam"[Mesh] OR "Diazepam"[Mesh] OR "Valproic Acid"[Mesh] OR "eslicarbazepine acetate" [Supplementary Concept] OR "Ethosuximide"[Mesh] OR "ezogabine" [Supplementary Concept] OR "felbamate" [Supplementary Concept] OR "gabapentin" [Supplementary Concept] OR "etiracetam" [Supplementary Concept] OR "lamotrigine" [Supplementary Concept] OR "lacosamide" [Supplementary Concept] OR "Lorazepam"[Mesh] OR "oxcarbamazepine" [Supplementary Concept] OR "perampanel" [Supplementary Concept] OR "Phenobarbital"[Mesh] OR "Phenytoin"[Mesh] OR "Pregabalin"[Mesh] OR "Primidone"[Mesh] OR "rufinamide" [Supplementary Concept] OR "tiagabine" [Supplementary Concept] OR "topiramate" [Supplementary Concept] OR "Vigabatrin"[Mesh] OR "zonisamide" [Supplementary Concept]
OR Seizures OR Epilepsy OR Anticonvulsants OR Barbiturates Propofol OR Hydantoins OR Carbamazepine OR brivaracetam OR clobazam OR Clonazepam OR Diazepam OR Valproic Acid OR eslicarbazepine acetate OR Ethosuximide OR ezogabine OR felbamate OR gabapentin OR etiracetam OR lamotrigine OR lacosamide OR Lorazepam OR oxcarbamazepine OR perampanel OR Phenobarbital OR Phenytoin OR Pregabalin OR Primidone OR rufinamide OR tiagabine OR topiramate OR Vigabatrin OR zonisamide) AND ("prevention and control" [Subheading] OR “Tertiary Prevention"[Mesh] OR "Secondary Prevention"[Mesh] OR "Primary Prevention"[Mesh] OR "Pre-Exposure Prophylaxis”[Mesh] OR “Post-Exposure Prophylaxis”[Mesh] OR prevention OR control OR prophylactic OR prophylaxis)

\section{Cochrane Central Register of Controlled Trials (CENTRAL)}

(Neurosurgery OR neurosurgical procedures OR general surgery OR operative surgical procedures OR surgery or brain surgery or craniectomy OR neurological surgery OR craniotomy OR operation OR operative OR procedure OR resection OR surgical or surgical procedure) AND (Brain Neoplasms OR brain tumor OR brain neoplasm) AND (Seizures OR Epilepsy OR Anticonvulsants OR Barbiturates OR Propofol OR Hydantoins OR Carbamazepine OR brivaracetam OR clobazam OR Clonazepam OR Diazepam OR Valproic Acid OR eslicarbazepine acetate OR Ethosuximide OR ezogabine OR felbamate OR gabapentin OR etiracetam OR lamotrigine OR lacosamide OR Lorazepam OR oxcarbamazepine OR perampanel OR Phenobarbital OR Phenytoin OR Pregabalin OR Primidone OR rufinamide OR tiagabine OR topiramate OR Vigabatrin OR zonisamide) AND (Prevention or control or prophylaxis or prophylactic)

\section{CINAHL/Academic Search Complete}

(Neurosurgery OR neurosurgical procedures OR general surgery OR operative surgical procedures OR surgery or brain surgery or craniectomy OR neurological surgery OR craniotomy OR operation OR operative OR procedure OR resection OR surgical or surgical procedure) AND (Brain Neoplasms OR brain tumor OR brain neoplasm) AND (Seizures OR Epilepsy OR Anticonvulsants OR Barbiturates OR Propofol OR Hydantoins OR Carbamazepine OR brivaracetam OR clobazam OR Clonazepam OR Diazepam OR Valproic Acid OR eslicarbazepine acetate OR Ethosuximide OR ezogabine OR felbamate OR gabapentin OR etiracetam OR lamotrigine OR lacosamide OR Lorazepam OR oxcarbamazepine OR perampanel OR Phenobarbital OR Phenytoin OR Pregabalin OR Primidone OR rufinamide OR tiagabine OR topiramate OR Vigabatrin OR zonisamide) AND (Prevention or control or prophylaxis or prophylactic)

\section{Web of Science}

(Neurosurgery OR neurosurgical procedures OR general surgery OR operative surgical procedures OR surgery or brain surgery or craniectomy OR neurological surgery OR craniotomy OR operation OR operative OR procedure OR resection OR surgical or surgical procedure) AND TOPIC: (Brain Neoplasms OR brain tumor OR brain neoplasm) AND TOPIC: (Seizures OR Epilepsy OR Anticonvulsants OR Barbiturates OR Propofol OR Hydantoins OR Carbamazepine OR brivaracetam OR clobazam OR Clonazepam OR Diazepam OR Valproic Acid OR eslicarbazepine acetate OR Ethosuximide OR ezogabine OR felbamate OR gabapentin OR etiracetam OR lamotrigine OR lacosamide OR Lorazepam OR oxcarbamazepine OR perampanel OR Phenobarbital OR Phenytoin OR Pregabalin OR Primidone OR rufinamide OR tiagabine OR topiramate OR Vigabatrin OR zonisamide) $A N D$ TOPIC: (Prevention or control or prophylaxis or prophylactic) AND TOPIC: (clinical trial OR comparative study OR multicenter study OR controlled clinical trial OR pragmatic clinical trial OR randomized controlled trial OR validation studies) 


\section{ScienceDirect}

(neurosurgery or neurosurgical procedure or operative or surgery or craniectomy or neurological surgery or craniotomy or operation or operative or procedure or resection or surgical) and (brain neoplasm or brain tumor or brain neoplasm) and (brain neoplasm or brain tumor or brain neoplasm) and (seizure or epilepsy or anticonvulsant or antiepileptic) and (prevention or prophylaxis or prophylactic) and (clinical trial OR comparative study OR multicenter study OR controlled clinical trial OR pragmatic clinical trial OR randomized controlled trial OR validation studies) AND human AND English

\section{Acknowledgments}

Services in support of the research project were generated by the VCU Massey Cancer Center Biostatistics Shared Resource, supported, in part, with funding from NIH-NCI Cancer Center Support Grant P30 CA016059.

\section{References}

1. Beenen LF, Lindeboom J, Kasteleijn-Nolst Trenité DG, Heimans JJ, Snoek FJ, Touw DJ, et al: Comparative double blind clinical trial of phenytoin and sodium valproate as anticonvulsant prophylaxis after craniotomy: efficacy, tolerability, and cognitive effects. J Neurol Neurosurg Psychiatry 67:474-480, 1999

2. De Santis A, Villani R, Sinisi M, Stocchetti N, Perucca E: Add-on phenytoin fails to prevent early seizures after surgery for supratentorial brain tumors: a randomized controlled study. Epilepsia 43:175-182, 2002

3. Dewan MC, Thompson RC, Kalkanis SN, Barker FG II, Hadjipanayis CG: Prophylactic antiepileptic drug administration following brain tumor resection: results of a recent AANS/CNS Section on Tumors survey. J Neurosurg 126:1772-1778, 2017

4. Dewan MC, White-Dzuro GA, Brinson PR, Thompson RC, Chambless LB: Perioperative seizure in patients with glioma is associated with longer hospitalization, higher readmission, and decreased overall survival. J Neurosurg 125:1033-1041, 2016

5. Dewan MC, White-Dzuro GA, Brinson PR, Zuckerman SL, Morone PJ, Thompson RC, et al: The influence of perioperative seizure prophylaxis on seizure rate and hospital quality metrics following glioma resection. Neurosurgery 80:563-570, 2017

6. Farrington CP, Manning G: Test statistics and sample size formulae for comparative binomial trials with null hypothesis of non-zero risk difference or non-unity relative risk. Stat Med 9:1447-1454, 1990

7. Forsyth PA, Weaver S, Fulton D, Brasher PM, Sutherland G, Stewart D, et al: Prophylactic anticonvulsants in patients with brain tumour. Can J Neurol Sci 30:106-112, 2003

8. Franceschetti S, Binelli S, Casazza M, Lodrini S, Panzica F, Pluchino F, et al: Influence of surgery and antiepileptic drugs on seizures symptomatic of cerebral tumours. Acta Neurochir (Wien) 103:47-51, 1990

9. Fuller KL, Wang YY, Cook MJ, Murphy MA, D’Souza WJ: Tolerability, safety, and side effects of levetiracetam versus phenytoin in intravenous and total prophylactic regimen among craniotomy patients: a prospective randomized study. Epilepsia 54:45-57, 2013

10. Higgins JP, Altman DG, Gøtzsche PC, Jüni P, Moher D, Oxman AD, et al: The Cochrane Collaboration's tool for assessing risk of bias in randomised trials. BMJ 343:d5928, 2011

11. Jennum P, Gyllenborg J, Kjellberg J: The social and economic consequences of epilepsy: a controlled national study. Epilepsia 52:949-956, 2011
12. Kazerooni R, Bounthavong M: Cost-effectiveness analysis of intravenous levetiracetam versus intravenous phenytoin for early onset seizure prophylaxis after neurosurgery and traumatic brain injury. Clinicoecon Outcomes Res 2:15-23, 2010

13. Kern K, Schebesch KM, Schlaier J, Hansen E, Feigl GC, Brawanski AT, et al: Levetiracetam compared to phenytoin for the prevention of postoperative seizures after craniotomy for intracranial tumours in patients without epilepsy. J Clin Neurosci 19:99-100, 2012

14. Kuijlen JM, Teernstra OP, Kessels AG, Herpers MJ, Beuls EA: Effectiveness of antiepileptic prophylaxis used with supratentorial craniotomies: a meta-analysis. Seizure 5:291298, 1996

15. Levati A, Savoia G, Zoppi F, Boselli L, Tommasino C: Peri-operative prophylaxis with phenytoin: dosage and therapeutic plasma levels. Acta Neurochir (Wien) 138:274279, 1996

16. Lim DA, Tarapore P, Chang E, Burt M, Chakalian L, Barbaro N, et al: Safety and feasibility of switching from phenytoin to levetiracetam monotherapy for glioma-related seizure control following craniotomy: a randomized phase II pilot study. J Neurooncol 93:349-354, 2009

17. Logan JK, Tang C, Liao Z, Lee JJ, Heymach JV, Swisher SG, et al: Analysis of factors affecting successful clinical trial enrollment in the context of three prospective, randomized, controlled trials. Int J Radiat Oncol Biol Phys 97:770-777, 2017

18. Moher D, Shamseer L, Clarke M, Ghersi D, Liberati A, Petticrew M, et al: Preferred reporting items for systematic review and meta-analysis protocols (PRISMA-P) 2015 statement. Syst Rev 4:1, 2015

19. Nakamura N, Ishijima B, Mayanagi Y, Manaka S: A randomized controlled trial of zonisamide in postoperative epilepsy: a report of the cooperative group study. Jpn J Neurosurg 8:647-656, 1999

20. North JB, Penhall RK, Hanieh A, Frewin DB, Taylor WB: Phenytoin and postoperative epilepsy. A double-blind study. J Neurosurg 58:672-677, 1983

21. Perucca E: Optimizing antiepileptic drug treatment in tumoral epilepsy. Epilepsia 54 (Suppl 9):97-104, 2013

22. Rosati A, Tomassini A, Pollo B, Ambrosi C, Schwarz A, Padovani A, et al: Epilepsy in cerebral glioma: timing of appearance and histological correlations. J Neurooncol 93:395-400, 2009

23. Shershever AS, Bentsion DL, Lavrova SA, Sorokova EV: [Use of Keppra during the radiotherapy in patients with brain tumors and epileptic seizures after surgical treatment.] Zh Nevrol Psikhiatr Im S S Korsakova 108:31-36, 2008 (Russian)

24. Sirven JI, Wingerchuk DM, Drazkowski JF, Lyons MK, Zimmerman RS: Seizure prophylaxis in patients with brain tumors: a meta-analysis. Mayo Clin Proc 79:1489-1494, 2004

25. Temkin NR: Antiepileptogenesis and seizure prevention trials with antiepileptic drugs: meta-analysis of controlled trials. Epilepsia 42:515-524, 2001

26. Türe H, Sayin M, Karlikaya G, Bingol CA, Aykac B, Türe $\mathrm{U}$ : The analgesic effect of gabapentin as a prophylactic anticonvulsant drug on postcraniotomy pain: a prospective randomized study. Anesth Analg 109:1625-1631, 2009

27. Wu AS, Trinh VT, Suki D, Graham S, Forman A, Weinberg JS, et al: A prospective randomized trial of perioperative seizure prophylaxis in patients with intraparenchymal brain tumors. J Neurosurg 118:873-883, 2013

28. Zadeghan SY, Sajedi P, Khalili GR: Comparing 'remifentanil-propofol' and 'fentanyl-propofol' in patients undergoing craniotomy for supratentorial space-occupying lesions. Tehran Univ Med J 70:548-554, 2012 


\section{Disclosures}

The authors report no conflict of interest concerning the materials or methods used in this study or the findings specified in this paper.

\section{Author Contributions}

Conception and design: Broaddus, Opalak, Stary. Acquisition of data: Rock, Carr. Analysis and interpretation of data: Rock,

Sima. Drafting the article: Chandra, Rock, Opalak, Carr. Critically revising the article: Broaddus, Chandra, Opalak. Reviewed submitted version of manuscript: Broaddus, Opalak, Sima, Vega. Approved the final version of the manuscript on behalf of all authors: Broaddus. Statistical analysis: Rock. Administrative/technical/material support: Broaddus. Study supervision: Broaddus.

\section{Correspondence}

William C. Broaddus, Department of Neurosurgery, Virginia Commonwealth University Health System, PO Box 980631, Richmond, VA 23219. email: william.broaddus@vcuhealth.org. 